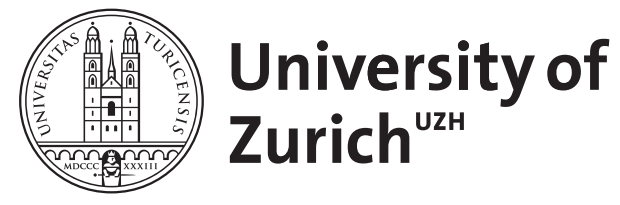

Zurich Open Repository and Archive

University of Zurich

University Library

Strickhofstrasse 39

CH-8057 Zurich

www.zora.uzh.ch

Year: 2013

\title{
Erfassung des Sturzrisikos
}

Neuner-Jehle, S

Posted at the Zurich Open Repository and Archive, University of Zurich ZORA URL: https://doi.org/10.5167/uzh-93675

Journal Article

Published Version

Originally published at:

Neuner-Jehle, S (2013). Erfassung des Sturzrisikos. PrimaryCare, 13(18):326. 


\section{Erfassung des Sturzrisikos}

Ein Beitrag aus der Reihe «Die Werkzeugkiste des Familienarztes»

Stürze gehören zu den wichtigen Ursachen von Krankheit, Behinderung und Tod im Alter. Die frühzeitige Erkennung eines erhöhten Sturzrisikos gefolgt von der Abklärung und Behandlung möglicher Ursachen (wie zum Beispiel Behandlung von Schwindelursachen, Verbesserung des Visus, Beseitigung von Rutschfallen etc.) ist also eine relevante Massnahme zur Sturzprävention in der Hausarztpraxis. Wie identifizieren wir rechtzeitig gefährdete Patienten, noch bevor sie durch gehäufte Stürze auffallen?

\section{Das Gangbild}

Ein schwankender, breitbeiniger Gang gilt als augenscheinliches Zeichen für die Gehunsicherheit und für ein erhöhtes Sturzrisiko. Nicht nur die Spurtreue, die mittels Strichgang erkennbar ist, sondern auch die Schrittlängen-Variabilität sind Indikatoren: Je unregelmässiger die Schrittlänge, desto höher ist das Sturzrisiko.

\section{«Timed up and go»-Test [1]}

Wie der Name sagt, wird die benötigte Zeit gemessen, um aufzustehen, drei Meter mit einer normalen (spontanen) Geschwindigkeit zu gehen, umzudrehen und sich wieder hinzusetzen. Unter 10 Sekunden bedeuten keine, 10 bis 20 Sekunden eine leichte, 20 bis 30 Sekunden eine moderate und über 30 Sekunden eine schwere Beeinträchtigung der Mobilität. Der Grenzwert für ein erhöhtes Sturzrisiko liegt bei 14 Sekunden. Gehhilfen dürfen benutzt werden. Obwohl ursprünglich an nur 60 Patienten validiert, hat der Test grosse Verbreitung gefunden und seine Nützlichkeit unter Beweis gestellt. Sein grosses Plus ist die rasche und einfache Durchführbarkeit.

\section{«Stops Walking When Talking»-Test [2]}

Müssen ältere Patienten zur Beantwortung einer einfachen Frage stehen bleiben, beispielsweise auf dem Weg vom Wartezimmer ins Sprechzimmer, dann bedeutet dies ein deutlich erhöhtes Sturzrisiko in den kommenden sechs Monaten. Und umgekehrt: Patienten, welche während des Gehens eine Frage beantworten können (Dual-Tasking), haben ein weitaus geringeres Sturzrisiko. Aber Achtung bei der Durchführung: Hüten Sie sich davor, selbst stehen zu bleiben und damit den Patien-ten auszubremsen. Dann sagt der
Test höchstens etwas über Sie selbst aus...

\section{Tinetti-Test, auch Perfor-} mance Orientated Mobility Assessment (POMA) [3]

Bereits eine ausführliche Sturzrisiko-Analyse, die ins Assessment eines Geriaters passt, aufgrund des Aufwandes aber weniger in die Sprechstunde des Hausarztes. Ein Punktescore, der die beiden Bereiche Gleichgewicht (im Sitzen, Stehen und Drehen) und Gangbild benotet. Für weniger als 20 von möglichen 28 Punkten ist das Sturzrisiko signifikant erhöht.

Link zum Tinetti-Test:

http://www.physioportal.ch/downloads/PDF/Tinetti\%20Test. pdf

Ich danke Yves Gschwind, PhD, Basel Mobility Center, Akutgeriatrische Universitätsklinik (AGUK), Universitätsspital Basel, für die kritische Durchsicht.

\section{Literatur}

1 Podsiadlo D, Richardson S. The timed «Up \& Go»: A test of basic functional mobility for frail elderly persons. J Am Geriatr Soc. 1991;39:142-8.

2 Lundin-Olsson L, Nyberg L, Gustafson Y. «Stops walking when talking» as a predictor of falls in elderly people. Lancet. 1997;349:617.

3 Tinetti ME. Performance-orientated assessment of mobility problems in elderly patients. J Am Geriatr Soc. 1986;34(2):119-26.

\section{Korrespondenz:}

Dr. med. Stefan Neuner-Jehle, MPH

Institut für Hausarztmedizin Zürich

Pestalozzistrasse 24

8091 Zürich

sneuner[at]bluewin.ch 